\title{
Hydration and Conformational Equilibrium in Yeast Thioredoxin 1: Implication for $\mathrm{H}^{+}$Exchange
}

\author{
Carolina Cruzeiro-Silva, ${ }^{\dagger}$ Francisco Gomes-Neto, ${ }^{\ddagger}$ Luciana E. S. F. Machado, ${ }^{\dagger}$ Catarina A. Miyamoto,
} Anderson S. Pinheiro, ${ }^{\S}$ Natalia Correa-Pereira, ${ }^{\dagger}$ Mariana T. Q. de Magalhães, ${ }^{\dagger}$ Ana Paula Valente, ${ }^{\dagger}$ and Fabio C. L. Almeida* ${ }^{*}$

${ }^{\dagger}$ Institute of Medical Biochemistry, National Center of Nuclear Magnetic Resonance Jiri Jonas, Federal University of Rio de
Janeiro-Institute of Structural Biology and Bioimaging, Rio de Janeiro, Brazil
${ }^{\ddagger}$ Laboratory of Toxinology, Instituto Oswaldo Cruz, Fiocruz, 21045-900 Rio de Janeiro, Brazil
${ }^{\S}$ Institute of Chemistry, Federal University of Rio de Janeiro, Rio de Janeiro, Brazil
${ }^{\|}$Faculdades Integradas de Três Lagoas, AEMS, Três Lagoas, Brazil

Supporting Information

ABSTRACT: One of the ancestral features of thioredoxins is the presence of a water cavity. Here, we report that a largely hydrated, conserved, buried aspartic acid in the water cavity modulates the dynamics of the interacting loops of yeast thioredoxin 1 (yTrx1). It is well-established that the aspartic acid, Asp24 for yTrx1, works as a proton acceptor in the reduction of the target protein. We propose a complementary role for Asp24 of coupling hydration and conformational motion of the water cavity and interacting loops. The intimate contact between the water cavity and the interacting loops means that motion at the water cavity will affect the interacting loops and vice versa. The D24N mutation alters the conformational equilibrium for both the oxidized and reduced states, quenching the conformational motion in the water cavity. By measuring the hydration and molecular dynamics simulation of wild-type yTrx1 and the D24N mutant, we showed that Asn24 is more exposed to water than Asp24 and the water cavity is smaller in the mutant, closing the inner part of the water cavity. We discuss how the conformational equilibrium contributes to the mechanism of catalysis and $\mathrm{H}^{+}$exchange.
$\mathrm{T}$ hioredoxins (Trx) are small proteins of approximately 105 residues, present in all organisms from Archea to the higher eukaryotes. Along with glutaredoxins, they act to maintain the reductive potential of the cells. Oxidized Trx is recycled in the cell by thioredoxin reductases, using the reductive equivalent of $\mathrm{NADPH}$ (via $\mathrm{FADH}$ ). This cycle maintains the reductive capacity of Trx within the cytoplasm or mitochondria. ${ }^{4-6}$

In higher eukaryotes, the Trx system controls the redox state of many cellular proteins, such as apoptosis specific kinase 1 (ASK1), thioredoxin inhibitory protein (TxNIP), ribonucleotide reductase, peroxiredoxins, and NF-kB, DNA synthesis, oxidative stress defense, redox signaling, and NO signaling. The Trx system is also involved in cancer, cardiovascular diseases, inflammation, aging, diabetes, and neurodegenerative diseases. ${ }^{7}$ Thioredoxins have been shown to be indispensable in many life forms. $^{8}$

Similarly, the yeast $\operatorname{Trx}(y \operatorname{Trx})$ system has multiple functions as a hydrogen donor for $3^{\prime}$-phosphoadenylsulfate (PAPS) reductase, ${ }^{4}$ peroxiredoxins, and many other compounds. In contrast to human Trx, in Saccharomyces cerevisiae there are two orthlogous cytoplasmic Trx: Trx1 and Trx2. Our group determined the solution structure of the reduced $\operatorname{Trx}^{9,10}$ and
Trx2. ${ }^{11}$ The crystallographic oxidized structures are also available. $^{12-14}$

Recently, it has been shown that the conformation of thioredoxins has been conserved for at least 4 billion years, since the early stage of life on earth. ${ }^{15}$ Indeed, structural studies have shown the fold of Trx to be very similar and conserved in all species. It comprises a five-stranded central $\beta$-sheet surrounded by four $\alpha$-helices. This central hydrophobic $\beta$ sheet is formed by three parallel strands, $\beta 1-\beta 3$, and two more strands, $\beta 4$ and $\beta 5$, that are antiparallel to $\beta 3$. The active site is the main interacting region and is composed of the loop between $\beta 2$ and $\alpha$-helix $2(\alpha 2)$, which contains the conserved WCGPC motif. ${ }^{10}$ It participates directly in the interaction with thioredoxin's several partners in the cell. ${ }^{16}$ There are three other target-interacting loops, characterized by the structures of several complexes that have been determined. They face the same surface of the active site loop and are the $\beta 3-\alpha 3, \alpha 3-\beta 4$, and $\beta 5-\alpha 4$ loops. The $\alpha 3-\beta 4$ loop contains the conserved cis-

Received: November 16, 2013

Revised: April 15, 2014

Published: April 16, 2014 
proline residue that is important for the catalytic activity of thioredoxins. ${ }^{17}$

Other conserved features of thioredoxins are the presence of a water cavity adjacent to the active site and the presence of interacting loops underneath the interacting surface. The central residue of the water cavity is a conserved buried aspartic acid, which is located at $\beta 2$, the central $\beta$-strand, near the center of mass of the protein. The $\mathrm{p} K_{\mathrm{a}}$ of the buried aspartic acid has been determined to be approximately 7.6 , which is near the optimal catalytic activity of thioredoxins in general. The internal negatively charged residue and the water cavity are stabilized by the presence of a conserved lysine (strand $\beta 3$ ) in the vicinity. The long lysine side chain creates a positively charged environment near the buried aspartic acid and extending up to the protein surface. ${ }^{18}$

The structure of yTrx1 in the reduced state ${ }^{10}$ is characterized by the active site containing the exposed Cys 30 and the internal Cys33, which faces the water cavity. The buried aspartic acid is Asp24, and the conserved lysine is Lys54. The interacting surface is formed by three loop regions of residues 56-59, 7073 , and $90-93(\beta 3-\alpha 3, \alpha 3-\beta 4$, and $\beta 5-\alpha 4$ loops, respectively). Pro73 is the cis-proline in the $\alpha 3-\beta 4$ loop.

In 1980, Kallis and Holmgren proposed the basis of the protein disulfide reduction mechanism. ${ }^{19}$ First, reduced Trx binds to a target protein. This binding is essential, and its necessity is attributed to the subangstrom positioning of the target disulfide. $^{20,21}$ The thiolate of the exposed cysteine (Cys30) makes the first nucleophilic attack, resulting in the formation of a transient mixed disulfide. Second, the buried cysteine (Cys33) is activated to become a thiolate, gaining nucleophilicity. One of the proposed activation mechanisms involves the transfer of a proton to the buried aspartate (Asp24), with the participation of a water molecule bridging the cysteine and the aspartate. ${ }^{1,18,22}$ Finally, the second nucleophilic attack of the thiolate of Cys33 breaks the mixed disulfide and generates both the oxidized $\operatorname{Trx}$ and the reduced target protein. $^{23}$ There is still considerable debate concerning this mechanism of activation of the buried cysteines. ${ }^{24-27}$ Next, we will discuss the proposed mechanisms for cysteine activation in thioredoxins.

The exposed cysteine (Cys30) has a $\mathrm{p} K_{\mathrm{a}}$ of approximately 7 , which is at least 1 unit lower than the $p K_{a}$ of a free cysteine. The most widely accepted mechanism for its increased nucleophilicity is the stabilization of the thiolate by the proximity of the microdipole of $\alpha$-helix 2, which points toward it. $^{28}$ An alternate proposal is proton sharing between the two cysteines, where a "hydrogen bond" interaction was observed in several structures, with a hydrogen located between the two sulfurs. $^{29}$

In contrast with the exposed cysteines, it has been determined that the buried cysteine shows a $\mathrm{p} K_{\mathrm{a}}$ that is at least 1 unit higher than that of the free amino acid $(>9),{ }^{1,30}$ resulting in poor nucleophilicity. To answer these questions, there are several hypotheses, which are experimentally challenging to investigate: (i) the previously mentioned solvent-mediated proton transfer, ${ }^{22}$ based on the observation of water near the aspartic acid of several X-ray structures and the possible bridging between the cysteine and aspartic acid; (ii) direct proton abstraction by the leaving group produced by the reaction; ${ }^{25}$ and (iii) a conformational change of the mixed disulfide, which suggests that in the presence of the target protein, new hydrogen bonds are formed between the buried and exposed cysteines and the buried cysteines and the conserved tryptophan. ${ }^{24}$ The network of hydrogen bonds would reduce the $\mathrm{p} K_{\mathrm{a}}$ of the mixed disulfide conformation. This mechanism explains the cysteine activation without the direct participation of the buried aspartic acid.

The high efficiency of Trx is still not completely understood. Trx is able to reduce its substrate $10^{3}$ times more efficiently than small molecules such as dithiothreitol (DTT). ${ }^{31}$ Its efficiency has been attributed to a chaperone-like activity that allows the assessment of buried disulfide bonds. ${ }^{32,33}$ The molecular recognition of the target protein by $\operatorname{Trx}$ is responsible for the chaperone-like activity, most likely related to the presence of a hydrophobic patch at the surface. Wiita and colleagues proposed that the active site of $\operatorname{Trx}$ regulates, through molecular recognition, the geometry of the target disulfide at a subangstrom level, making the catalysis very efficient. $^{20,21}$

Here, we report the dynamics of yTrxl and the D24N mutant. Trxl shows conformational exchange at the interacting loops and at the water cavity, which contains buried aspartic acid Asp24. We showed that replacing Asp24 with Asn modulates complex motions involving the catalytic loop and other interacting loops. We hypothesize that the Asn residue mimics the protonated form of Asp and that the hydration and conformational equilibrium involving this residue control access to the water cavity. The dynamics studies of the D24N mutant showed that the mutations stabilized a conformational state, quenching most of the conformational exchange at the water cavity. To correlate the milli to microsecond dynamics with the hydration of residue 24 , we measured the rotating frame and laboratory frame nuclear Overhauser effects (NOEs). We probed the presence of dipolar interaction between Asp24 and water for yTrx1 but not for D24N. Molecular dynamics (MD) simulations showed that yTrxl, in its oxidized and reduced forms, had a long-lived water molecule hydrogen-bonded with Asp24, while there were only short-lived hydrogen bonds $(<1$ ns) for all exposed carboxyl groups in the protein and also with $\mathrm{N} 24$ in the D24N mutant.

\section{METHODOLOGY}

Expression and Purification of Yeast Thioredoxin 1. The transformation was performed in Escherichia coli BL21(DE3) pLysS using plasmid pET17-b containing the sequence of yTrx1 or the D24N mutant. The cells were grown to an $\mathrm{OD}_{600}$ of 0.7 in $\mathrm{M} 9$ containing ${ }^{15} \mathrm{NH}_{4} \mathrm{Cl}$ and induced with 1 $\mathrm{mM}$ isopropyl $\beta$-D-1-thiogalactopyranoside for $5 \mathrm{~h}$. The cells were centrifuged, and the pellets were resuspended in $20 \mathrm{mM}$ $\mathrm{NaH}_{2} \mathrm{PO}_{4}$ (pH 7), $10 \mathrm{mM} \beta$-mercaptoethanol, and $40 \mu \mathrm{g} / \mathrm{mL}$ phenylmethanesulfonyl fluoride and lysed by sonication. After centrifugation, the supernatant was purified in exchange-ion QSepharose with a salt gradient and by size exclusion on Sephacryl 26/60 with $20 \mathrm{mM} \mathrm{NaH} \mathrm{PO}_{4}(\mathrm{pH}$ 7). The protein purity was confirmed by sodium dodecyl sulfate-polyacrylamide gel electrophoresis.

NMR Experiments. NMR spectra were recorded on 600 $\mathrm{MHz}$ Bruker DRX and $800 \mathrm{MHz}$ Bruker Avance III instruments at $25{ }^{\circ} \mathrm{C}$. Topspin version 2.1 (Bruker Biospin) was used to acquire the data. The NMR spectra were processed using NMRPipe version $2.3^{34}$ and analyzed using NMR ViewJ. ${ }^{35}$ All the data were collected with $200 \mu \mathrm{M}$ wild-type (wt) $\left.{ }^{15} \mathrm{~N}\right] \operatorname{Trx} 1$ and mutant D24N $\left[{ }^{15} \mathrm{~N}\right] \operatorname{Trx} 1$ in the oxidized and reduced states. The protein was reduced via the addition of $30 \mathrm{mM}$ deuterium DTT to the sample. 
Relaxation Experiments. The dynamic experiments were analyzed by ${ }^{15} \mathrm{~N} R_{1},{ }^{15} \mathrm{~N} R_{2}$, and ${ }^{1} \mathrm{H}-{ }^{15} \mathrm{~N}$ heteronuclear $\mathrm{NOE}$ with 1024 and 96 complex points in $F_{2}\left({ }^{1} \mathrm{H}\right)$ and $F_{1}\left({ }^{15} \mathrm{~N}\right)$, respectively. $R_{1}$ and $R_{2}$ values were collected with 11 accumulations by increment and ${ }^{1} \mathrm{H}-{ }^{15} \mathrm{~N}$ NOE values with 16 accumulations by increment. $R_{1}$ was measured with delays of $20,50,70,100,150,300,500,1000$, and $1500 \mathrm{~ms} . R_{2}$ was measured with delays of $47,136,78.56,109.984,141.408$, $172.832,204.256,235.68,267.104$, and $298.528 \mathrm{~ms} . R_{1}$ and $R_{2}$ values were collected three times at 100 and $78.56 \mathrm{~ms}$, respectively, to calculate the standard deviation. The ${ }^{1} \mathrm{H}-{ }^{15} \mathrm{~N}$ NOEs were acquired with or without proton saturation for $5 \mathrm{~s}$. $R_{1}$ and $R_{2}$ values were obtained using NMR View version 5.0. ${ }^{35}$ The ${ }^{1} \mathrm{H}-{ }^{15} \mathrm{~N}$ NOE values were determined using the intensity saturation spectra/intensity without saturation spectra ratio. The relaxation parameters were adjusted in accordance with Lipari-Szabo formalism ${ }^{36,37}$ to yield the intramolecular dynamic for each residue. To obtain the rotational correlation time $\left(\tau_{c}\right)$, the $R_{2} / R_{1}$ ratio was calculated and a Monte Carlo simulation was used. The relaxation parameters were fit according to the Lipari-Szabo model-free formalism using symmetric anisotropic diffusion tensors with Tensor2. ${ }^{38}$ The deposited structures of reduced and oxidized yTrx1 were used to calculate the diffusion tensors. ${ }^{10,13}$

Dipolar Interaction with Water. The NOESY-HSQC and ROESY-HSQC spectra were used to obtain the NOE at the laboratory and the rotating frame (ROE), respectively, of residues of wt $\left[{ }^{15} \mathrm{~N}\right] \mathrm{y} \operatorname{Trx} 1$ or mutant D24N $\left[{ }^{15} \mathrm{~N}\right] \mathrm{yTrx} 1$ with water. These data were acquired with 1024, 32, and 64 complex points in $F_{3}\left({ }^{1} \mathrm{H}\right), F_{2}\left({ }^{15} \mathrm{~N}\right)$, and $F_{1}\left({ }^{1} \mathrm{H}\right)$, respectively, using 16 scans and a mixing time of $20 \mathrm{~ms}$.

Mapping of Structural Change. The changes in the structure of mutant D24N Trx1 compared with that of wt Trx1 were mapped by measuring the chemical shift difference in the ${ }^{1} \mathrm{H}-{ }^{15} \mathrm{~N}$ HSQC spectra. The $400 \mu \mathrm{M}$ sample was used in the oxidized and reduced state for both the mutant and wt Trx1. The chemical shift difference between the amide hydrogen and nitrogen was quantified using the following equation:

$$
\Delta \delta=\left[\left(\Delta^{\mathrm{HN}}\right)^{2}+\left(\frac{\Delta^{\mathrm{N}}}{10}\right)^{2}\right]^{1 / 2}
$$

where $\Delta^{\mathrm{HN}}$ and $\Delta^{\mathrm{N}}$ are the chemical shift differences, in parts per million, observed for the hydrogen and nitrogen nuclei, respectively.

Molecular Dynamics (MD) Simulation. To identify the hydration effect of the Asp24 residue in the yTrx1 dynamics, we performed two pairs of $200 \mathrm{~ns}$ molecular dynamics simulations. Each pair is composed of wt Trxl (in the reduced or oxidized form) and the corresponding D24N mutant.

Starting Structures. The simulation of wt Trx1 in the reduced form was begun using the first structure in the NMR solution structure of Protein Data Bank (PDB) entry $2 \mathrm{I}^{2} \mathrm{H}^{10}$ To simulate the oxidized state (wt), we used the crystal structure of PDB entry 3F3Q. ${ }^{13}$ The ionization states of the side chains and the $\mathrm{N}$ - and $\mathrm{C}$-termini were calculated with PROPKA using $\mathrm{pH} 7.0 .^{39}$ For the wt reduced state of yTrxl, we decided to use the ionized form of Asp24 because of its effect on the mechanism of activation. The calculated $\mathrm{p} K_{\mathrm{a}}$ for Asp24 was 7.31 .

The D24N mutant structural models (reduced and oxidized forms) were generated using the molecular visualization program VMD-XPLOR ${ }^{40}$ by applying the "Mutate residue" module.

General MD Setup. All the MD simulations were conducted with GROMACS version $4.5 .4^{41}$ and via application of periodic boundary conditions. Long-range electrostatics were modeled using the particle mesh Ewald method (PME). ${ }^{42}$ Short-range repulsive/attractive dispersion interactions were modeled using the Lennard-Jones potential with a $1.0 \mathrm{~nm}$ cutoff for the real-space calculation. ${ }^{43}$ The LINCS $^{44}$ and SETTLE ${ }^{45}$ algorithms were used to constrain the bond lengths, allowing an integration time step of 2 fs. All simulations were coupled to an isotropic pressure bath of $1 \mathrm{~atm}$ and a heat bath of $298 \mathrm{~K}$ using standard coupling schemes.

The Amber99sb force field parameters were used to set up the molecular topologies. ${ }^{46}$ Water was modeled using the TIP3P model. ${ }^{47}$ To neutralize the system charges, sodium and chloride ions were inserted into the water solution.

The initial models (protein with water and ions) were subjected to an energy minimization procedure using three steps: hard position restrained steepest descents (harmonic constant of $1000 \mathrm{~kJ} \mathrm{~mol}^{-1} \mathrm{~nm}^{-2}$ ), steepest descents, and the conjugate gradient method to correct bad contacts and geometry deviations.

Next, using the GROMACS simulated annealing schedule, we performed a gradual increase in temperature, from 5 to 298 $\mathrm{K}$, under a hard position restraint potential on the protein heavy atoms, allowing water solvation. Sequentially, the position restraint potential was reduced stepwise from 1000 to $0 \mathrm{~kJ} \mathrm{~mol}^{-1} \mathrm{~nm}^{-2}$ in steps of $200 \mathrm{ps}$. Finally, the system was equilibrated in a $1 \mathrm{~ns} \mathrm{MD}$ simulation in which the root-meansquare deviation stabilized at $1 \pm 0.2 \AA$ for the backbone and 2 $\pm 0.2 \AA$ for side chains.

MD Simulation Analysis. The generated trajectories were analyzed using GROMACS version 4.5.4. All the images were generated using VMD.

Radial Distribution Function (RDF) of Water Molecules as a Function of Asp/Asn $g_{\text {SolAA }}(r)$. The analysis of the interactions of aspartic acid 24 (or asparagine 24) with water was performed by calculating the radial distribution functions (RDFs), $g(r)$, between -O/N side chain atom of the amino acid residue at position 24 of the Trx1 primary sequence and the water molecules.

Hydrogen Bond Analysis. The hydrogen bond analysis between Asp/Asn24 and the water molecules was performed using the geometrical criterion of a distance of $<0.35 \mathrm{~nm}$ and an angle of $<30^{\circ}$ applied in donor-hydrogen-acceptor atom triplets.

An H-bond existence map was generated (not shown), in which each detected hydrogen bond is identified by a unique (and sequential) numerical index and plotted as a function of simulation time. To evaluate the duration of the existence of each hydrogen bond, we counted the red pixels in the map and expressed the result as a function of a percentage of the total frames in the simulation. The percent hydrogen bond lifetime $(t)$ was classified into six arbitrary time ranges: 1-2.9, 3-6.9, $7-10.9,11-14.9,15-19$, and $>19$ ns.

Cavity Detection in Trx1. We used 20000 total structures extracted from the MD simulation trajectories for each model. Cavity detection was conducted using the Fpocket program suite based on Voronoi tessellation and $\alpha$-sphere detection. ${ }^{48}$ The structures were aligned and analyzed using MDPocket.

Fpocket $^{48}$ allows the observation of cavities in MD trajectories. It uses the concept of $\alpha$-spheres, which are spheres 


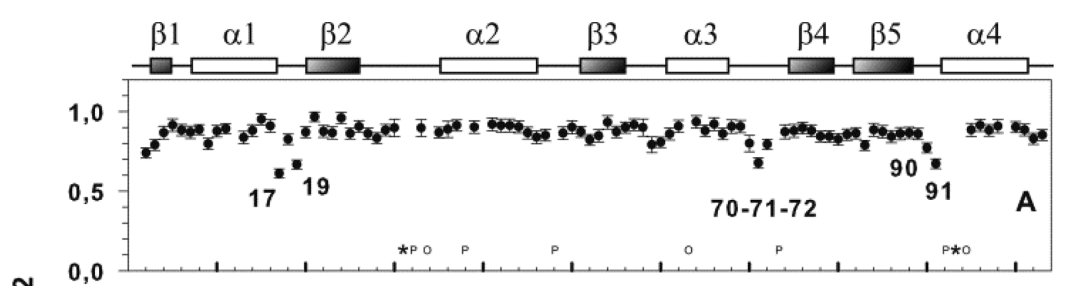

ஸู
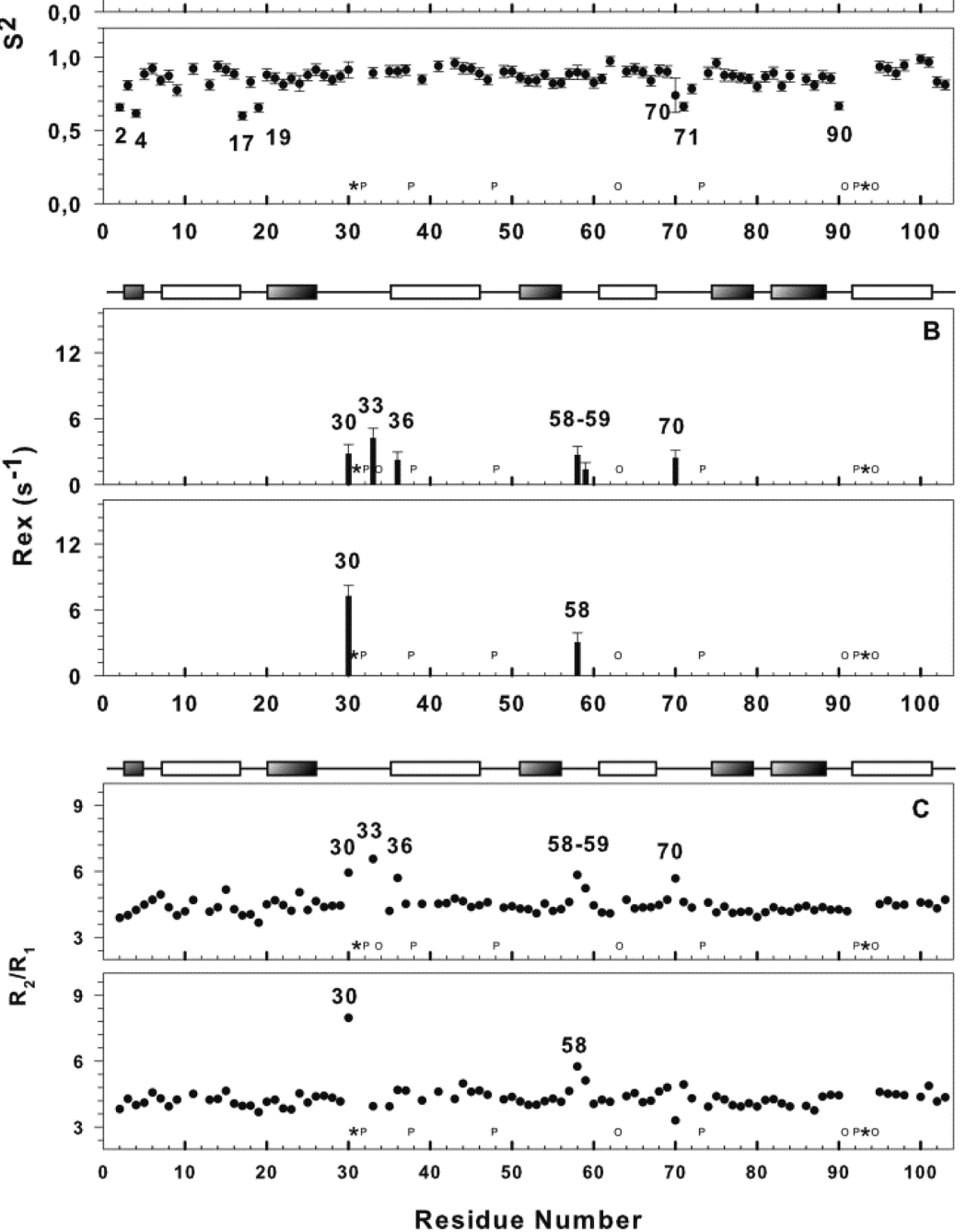

Figure 1. Dynamics of reduced yTrx1 fit according to the Lipari-Szabo model-free formalism. (A) Order parameter $\left(S^{2}\right)$ as a function of residue number of wt yTrx1 (top) and its D24N mutant (bottom). (B) Conformational exchange rate $\left(R_{\mathrm{ex}}\right)$ as a function of the residue number of wt yTrx1 (top) and its D24N mutant (bottom). The $R_{2} / R_{1}$ ratio as a function of the residue number of wt yTrxl (top) and its D24N mutant (bottom). Both yTrx1 and D24N were at a concentration of $200 \mu \mathrm{M}$ in $20 \mathrm{mM}$ phosphate buffer (pH 7.0) with $30 \mathrm{mM}$ perdeuterated DTT. The residues that could not be analyzed are denoted with empty circles for overlaps, asterisks for absent resonances due to line broadening, and P for prolines.

that contact four atoms in their boundary that are at equal distances. $\alpha$-Sphere radii reflect the compactness of the atoms and the curvature of a surface or cavity. Small $\alpha$-spheres are located at the protein core, while large ones are located at the surface. The software filters an ensemble of $\alpha$-spheres on a MD trajectory, allowing the location of intermediate size $\alpha$-spheres, which are typical of cavities. In this way, it is possible to define cavities that are representative of a MD trajectory.

After the overall analysis, cavity selection was performed by changing the isovalue in the VMD isosurface representation until different pockets were detected as isolated entities. A PDB file was generated containing the coordinates of the selected cavity and used to determine the volume changes as a function of $\mathrm{MD}$ simulation frame and the general features of this cavity.
All images were generated using VMD-Xplor with an isovalue of 2.2 and the cavities represented as solid isosurfaces.

\section{RESULTS}

In this work, we aim to understand the correlation between the dynamics of $y \operatorname{Trx} 1$ and its function. We measured the relaxation parameters of wt yeast thioredoxin 1 (yTrx1) and its D24N mutant $(\mathrm{D} 24 \mathrm{~N})$ in the reduced and oxidized states. The measurements were all performed at $200 \mu \mathrm{M}$, a concentration at which we determined that yTrx1 is monomeric. We determined that $y \operatorname{Trx} 1$ shows partial dimerization at concentrations of $>200 \mu \mathrm{M}$, leading to changes in the chemical shifts and relaxation parameters. A higher concentration particularly affects the slow dynamics, which are very important for this work. The assignment of the amidic resonances of the wt and 


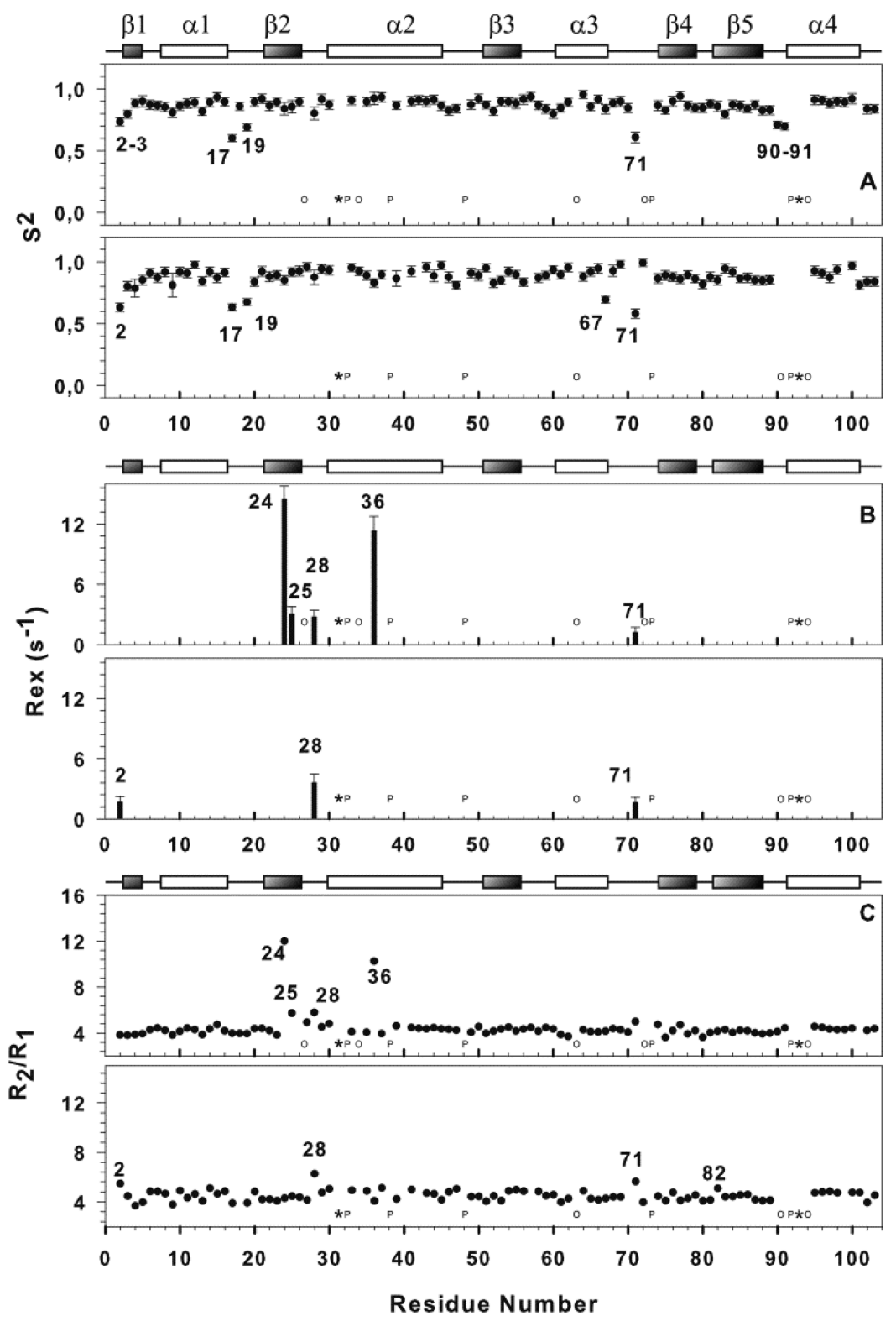

Figure 2. Dynamics of oxidized yTrx1 fit according to the Lipari-Szabo model-free formalism. (A) Order parameter $\left(S^{2}\right)$ as a function of the residue number of wt yTrxl (top) and its mutant D24N (bottom). (B) Conformational exchange rate $\left(R_{\mathrm{ex}}\right)$ as a function of the residue number of wt yTrx1 (top) and its D24N mutant (bottom). (C) $R_{2} / R_{1}$ ratio as a function of the residue number of wt yTrx1 (top) and its D24N mutant (bottom). Both yTrx1 and D24N were at a concentration of $200 \mu \mathrm{M}$ in $20 \mathrm{mM}$ phosphate buffer (pH 7.0). The residues that could not be analyzed are denoted with empty circles for overlaps, asterisks for absent resonances due to line broadening, and $\mathrm{P}$ for prolines.

mutant was based on previously published data. ${ }^{9,10}$ The amidic resonances of $\mathrm{G} 31, \mathrm{~K} 34$, and $\mathrm{A} 93$ could not be detected on the ${ }^{15} \mathrm{~N}$ HSQC spectra because of extensive line broadening. We could not observe the N-terminal M1.

We measured the longitudinal and transverse relaxation rates $\left(R_{1}\right.$ and $R_{2}$, respectively) and heteronuclear NOEs at $14.7 \mathrm{~T}$ (Figures S1-S3 of the Supporting Information). The data were fit according to the Lipari-Szabo model-free approach. ${ }^{36,37}$ Figures 1 and 2 show the order parameter $\left(S^{2}\right)$ and dynamical exchange contribution to $R_{2}\left(R_{\mathrm{ex}}\right)$ as a function of residue number for reduced and oxidized wt yTrx1 and its D24N mutant.

As expected for a well-folded globular protein, yTrxl is wellbehaved, with most residues showing order parameters of approximately 0.9 for both wt yTrx1 and its D24N mutant, for the oxidized and reduced forms. We observed reduced order parameters, indicating thermal flexibility, for the N-terminus; residues Gln17 and Lys19, which are part of the loop between $\alpha$-helix 1 and $\beta$-strand 2 ( $\alpha 1-\beta 2$ loop); residue Ala71, which is part of the $\alpha 3-\beta 4$ loop; and Ala90 and Asn91, which are part of the $\beta 5-\alpha 4$ loop. The $\alpha 3-\beta 4$ and $\beta 5-\alpha 4$ loops are known to participate in interactions with other targets, ${ }^{27,49}$ while the $\alpha 1-\beta 2$ loop is on the opposite side of the interaction surface. Similar behavior was observed for yTrx1 in the oxidized state. Thermal flexibility exists in the same regions for the oxidized and reduced states. The same was observed for the mutant D24N, with almost no change in thermal flexibility when compared with that of wt yTrxl.

The absence of thermal flexibility in the active site loop $(\beta 1-\alpha 2$ loop $)$ is remarkable. In the oxidized state, helix 2 spans residues 30-46, making the active site loop shorter. In the reduced form, helix 2 is broken at Cys33. This lack of motion 


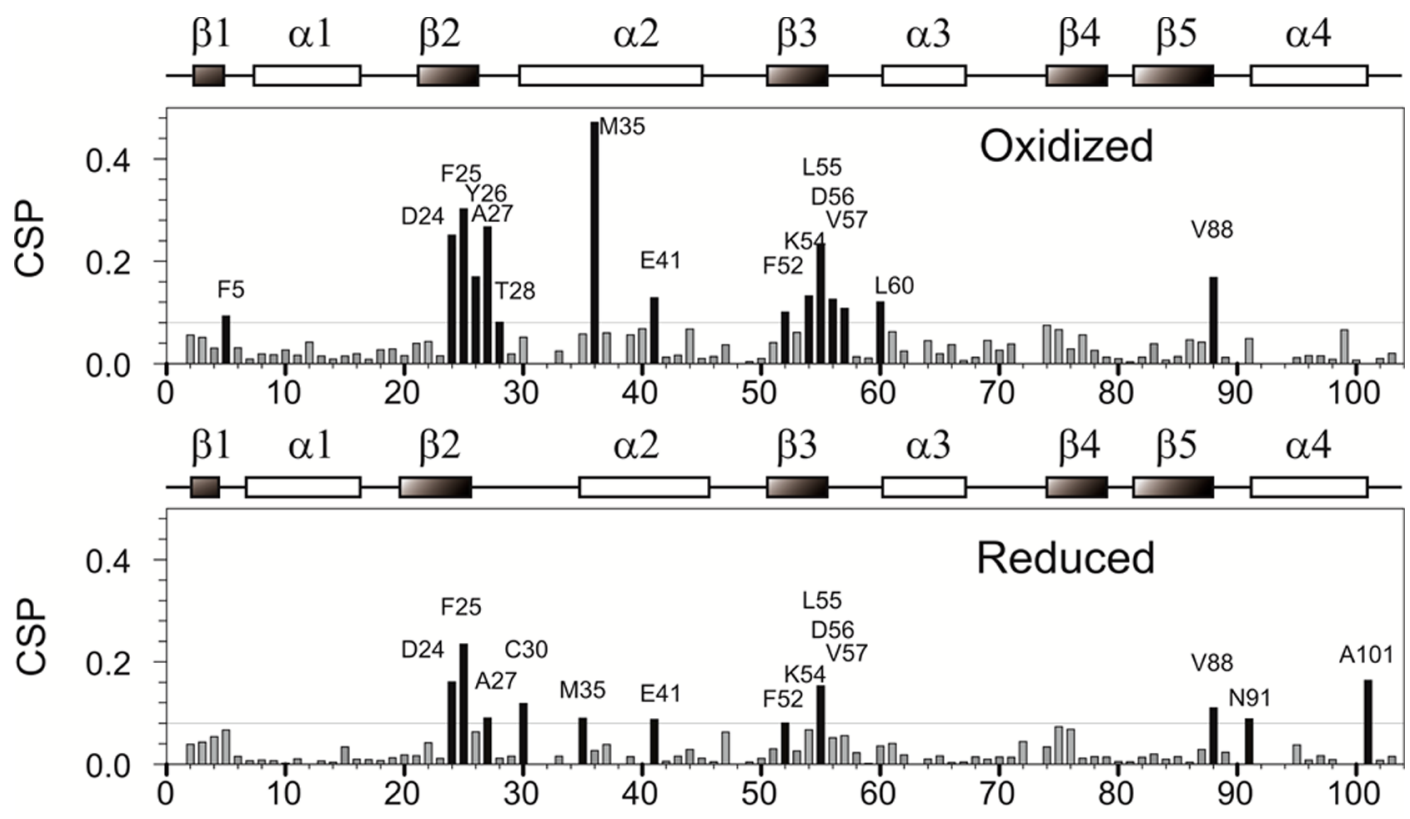

\section{Residue Number}
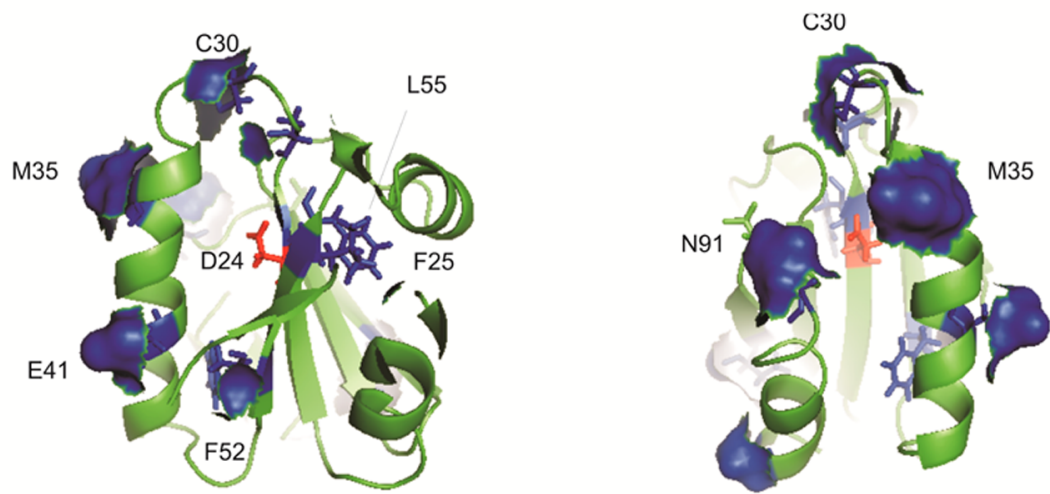

Figure 3. Chemical shift perturbation (CSP) between wt Trx1 and its D24N mutant in oxidized and reduced states. The top graphs show the CSP as a function of residue number. In the bottom panel, the ribbon representation of the yTrx1 structure illustrates CSP changes in the structure. Note that most chemical shift changes are more pronounced in the secondary structure elements, with emphasis on $\beta 1, \beta 2, \alpha 2, \beta 3$, and $\beta 4$. The black bars highlight the most pronounced changes, which were colored blue in the ribbon representation of the $y \operatorname{Trx} 1$ structure.

on the pico- to nanosecond time scale means that these three loops are well-structured and involved in conformational exchange, as we will discuss below.

The most striking difference among the conditions evaluated was observed for the micro- to millisecond dynamics. We observed conformational exchange for yTrxl in the reduced and oxidized conformations. We observed two sets of residues involved in conformational exchange: (i) residues that are part of the conserved interacting loops and also facing the solvent and (ii) residues that face the core of the protein and are exposed to the water cavity containing the well-described tightly bound water that bridges Cys33 and Asp24.

For the reduced conformation of $y \operatorname{Trx} 1, \mathrm{Cys} 30$ at the active site loop, Asp58 and Glu59 at the $\beta 2-\alpha 3$ loop, and Ser70 at the $\alpha 3-\beta 3$ interacting loop participate in the interacting loops and are in conformational exchange (Figure 1). Residues Cys33 and Ile36 face the water cavity.

The D24N mutation quenched the slow dynamics of the residues involved in the water cavity. It also increased the $R_{\mathrm{ex}}$ observed for Cys30. We also plotted the $R_{2} / R_{1}$ ratio for Trx 1 and D24N (Figure 1C). This parameter is independent of any fitting or physical model, and it is very easy to note the increase in the $R_{2} / R_{1}$ ratio for the residues described to be in conformational exchange.

For the oxidized conformation of yTrx1, Thr28 at the active site loop and Met71 at the $\alpha 3-\beta 3$ interacting loop belong to interacting loops and are in conformational exchange (Figure 2). Residues Asp24 and Ile36 are in the water cavity and are also in conformational exchange. Here again, the D24N mutation quenched the conformational exchange of the residues involved in the water cavity. Note that Asn24 and Ile36 are not in conformational exchange while Thr28 and Met71 remain with a positive value of $R_{\mathrm{ex}}$.

Gly31 and Ala93 could not be observed in the HSQC spectra, for the reduced or oxidized state, for the wt and mutant. They are line broadened because of intermediate conformational exchange, most likely due to dynamics of the adjacent prolines (Pro32 and Pro92). This information is 
important because the water cavity is surrounded by a cluster edged with four prolines (Figure S4 of the Supporting Information). Pro32 is at the active site loop at the beginning of helix 2; Pro38, which is highly conserved, is located in the middle of helix 2 . Pro73 is conserved for all thioredoxins and is located in the $\alpha 3-\beta 4$ interacting loop. Pro92 is in the $\beta 5-\alpha 4$ interacting loop. While Pro73 is in the cis conformation, all other prolines are predominantly trans. The cis-trans isomerization and ring puckering might play an important role in the dynamics of thioredoxin's interacting loops and water cavity. In particular, the dynamics of helix 2 are particularly important for the exchange of water in the cavity. The two highly conserved prolines, Pro32 and Pro38, might make an important contribution. Proline cis-trans isomerization and ring puckering contribute significantly to protein energetics. ${ }^{50}$ Endoexo puckering is isoenergetic for trans-prolines, but the exo pucker is much more prevalent for cis-prolines, stabilizing one specific configuration. This stabilization is particularly important for Pro73. Pro73 faces the water cavity and is part of an important interacting loop.

Figure 3 shows the chemical shift perturbation elicited by the D24N mutation, and Figure 4 illustrates the location of the

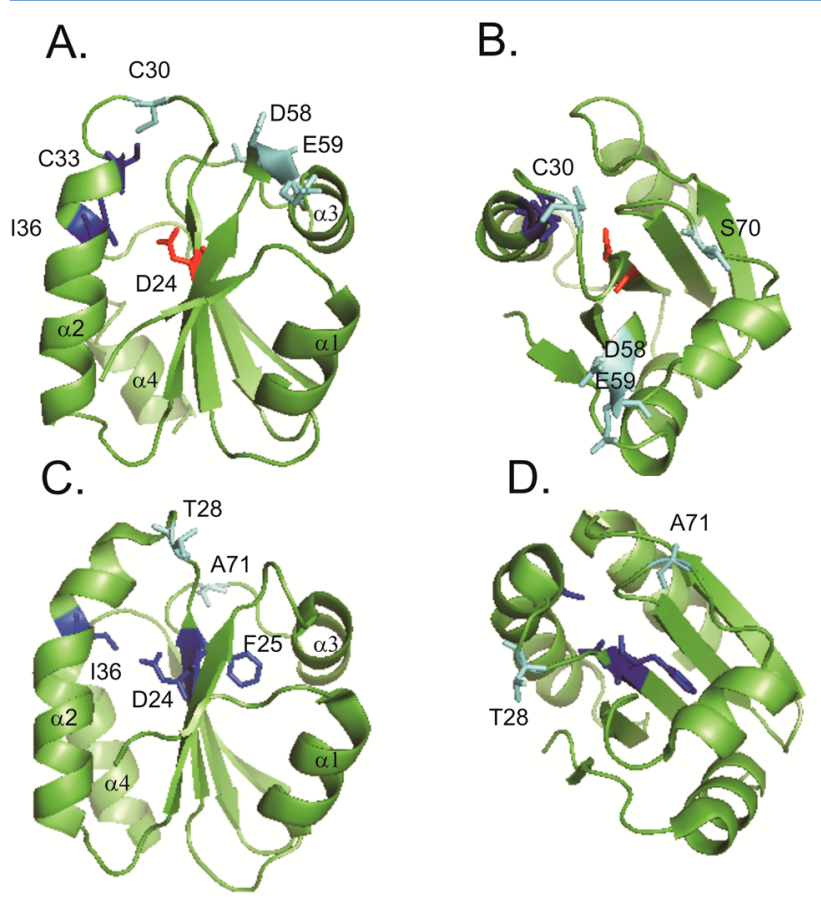

Figure 4. Summary of the residues in conformational exchange with yTrx1. Ribbon representations of yTrx1. Panels A and B show yTrx1 in the reduced state and panels $\mathrm{C}$ and $\mathrm{D} y \operatorname{Trx} 1$ in the oxidized state. Panels $A$ and $C$ are side views and panels $B$ and $D$ top views. In the side view (left), the interacting loops are at the top. The residues colored blue are the residues in conformational exchange, but these residues have a different chemical shift to share with the mutant.

residues in conformational exchange. The main chemical shift changes were observed in the central $\beta$-sheet, with the major changes at the top region of parallel strands $\beta 2$ and $\beta 3$ and in $\alpha$ helix 2, at residues Met35 and Glu41. These regions are the secondary structure elements that face the water cavity. On the other hand, the chemical shifts of the residues at the two $\alpha$ helices facing the other side of the $\beta$-sheet, which is opposite the water cavity ( $\alpha 1$ and $\alpha 3$ ), barely changed.
Asp24/Asn24 is in the middle of $\beta 2$, and the chemical shift changes in the residues of $\beta 3$ were expected. However, we observed only minor changes in $\beta 1$, which is also hydrogen bonded with $\beta 3$. There were minor chemical shift changes throughout the hydrophobic core of the protein, indicating a hydrophobic rearrangement. The chemical shift perturbations observed for the oxidized form are more prominent, but in general, the same residues are involved.

As mentioned above, the D24N mutation suppressed the conformational exchange of the residues facing the water cavity for the reduced and oxidized states. These data, along with the chemical shift perturbation elicited by the mutation, indicate that the D24N mutant, which contains Asn24 at the water cavity, stabilizes a conformational state, altering the condition in which water exchange is accomplished for yTrx1 (Figure 4). We hypothesized that the mutation affects the hydration of yTrxl through an opening-closing equilibrium of the water cavity.

Asp24/Asn24 modulates the slow motion of the interacting loops, indicating that there is a correlation between the solvation of the buried polar residues (Asp24/Asn24 and Lys54) and the dynamics of the interacting loops. Note that residue Cys30, which is responsible for the first nucleophilic attack on the target disulfide during the catalytic cycle, shows an $R_{\mathrm{ex}}$ of $\sim 3 \mathrm{~s}^{-1}$ for the wt and $\sim 8 \mathrm{~s}^{-1}$ for the mutant. These data suggest that Asp24 not only is a proton acceptor for the protein during the catalytic cycle but also is very important for the modulation of the thioredoxin dynamics, especially with regard to the dynamics of the water cavity. The hydration state of the water cavity is associated with the dynamics of the interacting loops. To experimentally probe this hypothesis, we designed experiments to improve our understanding of the correlation between the dynamics and hydration of Asp24/ Asn24.

It is not easy to probe protein hydration experimentally. In this research, we used multiple experiments that provide complementary information. One way to study hydration is to directly measure by NMR the dipolar coupling between water and protein hydrogens. This method was first proposed by Otting et al. ${ }^{51,52}$ It consists of measuring nuclear Overhauser correlations in the laboratory frame (NOE) and in the rotating frame (ROE). A negative NOE/ROE intensity ratio reveals interaction between the water and the protein residue. It was verified later that nonlocal water molecules in fast exchange with the interacting water molecule also contribute to the negative ratio. ${ }^{53}$ As a consequence, the presence of a negative ratio is not in itself conclusive with regard to the presence of a local tightly bound water. Thousands of water molecules can contribute to the negative $\mathrm{NOE} / \mathrm{ROE}$ intensity ratio. The method is valid only for internal water molecules that are motionally retarded. Luckily, this case holds for thioredoxins, in which tightly bound water is found in the water cavity, hydrogen-bonded to the buried aspartic acid. Most of the crystal structures of thioredoxins are of the oxidized state, and for all of them, tightly bound water is observed. There are fewer crystal structures for the reduced state, but the available crystal structures for reduced thioredoxins do show tightly bound water in the water cavity, hydrogen-bonded to Asp24 (PDB entries 2OE1, 4AJ6, and 3M9J)..$^{5-56}$ It is important to note that the use of heavy metals was not reported in the crystallization of these proteins. Heavy metals bind to the active site of the reduced thioredoxins. In conclusion, the 

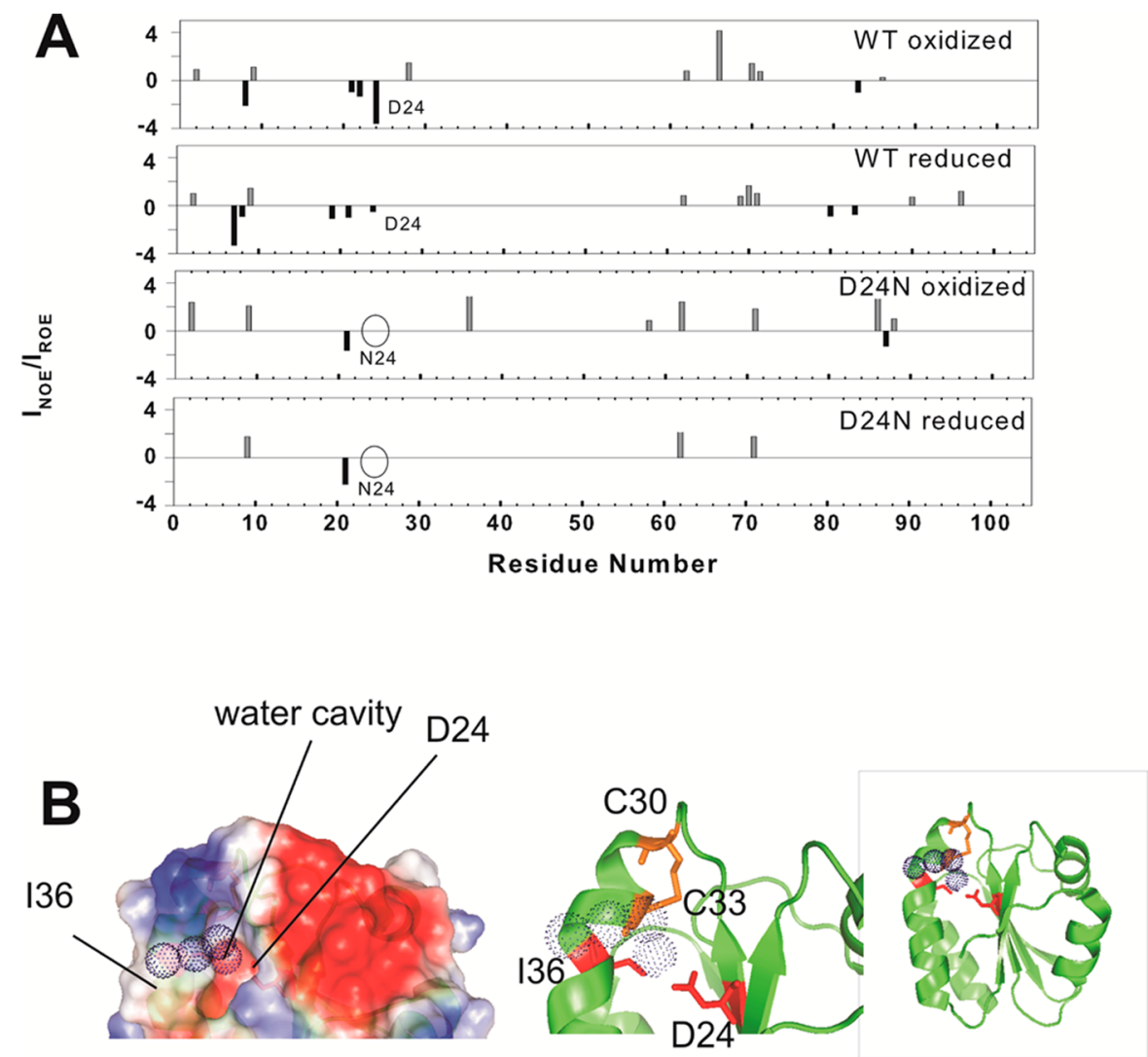

Figure 5. Hydration of wt and D24N y Trx1 in the oxidized and reduced states. (A) The nuclear Overhauser effects in the laboratory (NOE) and in the rotating frame (ROE) were measured with a mixing time of $20 \mathrm{~ms}$. The NOE/ROE ratio was plotted as a function of amino acid residue. A negative ratio represents the nuclear spin dipolar interaction between water and the $\mathrm{HN}$ for each amino acid. ${ }^{51}$ By using this method, local water interaction can be measured only for motionally retarded water molecules. ${ }^{57}$ This case holds for Asp24, where it is known for all X-ray structures of thioredoxins in the presence of tightly bound water. ${ }^{13,14}$ (B) X-ray structure of oxidized yTrxl. On the left, the electrostatic potential surface plot is shown, highlighting the presence of the water cavity, with tightly bound water in the cavity and a line of water molecules (blue dotted spheres, right).

tightly bound water at the cavity is a conserved feature present in all species studied so far.

Figure 5 shows the NOE/ROE ratio observed for yTrx 1 . We observed a few residues displaying negative ratios, but only for Asp24 can the effect be interpreted as being due to the local interaction of one motionally retarded water molecule or a few. Figure 5B shows the tightly bound water found near Asp24 in the water cavity. ${ }^{13}$ Note that there is a line of water molecules in the crystal. No crystal structure for the reduced form is available. Our NMR data confirm the presence of tightly bound water. We observed NOE and ROE cross-peaks with water for the oxidized and reduced conformations. The NOE/ROE ratio is more negative for the oxidized form, which could be interpreted as the water being more motionally retarded than in the reduced state. ${ }^{51,52}$ In this work, we prefer not to overinterpret these data because it depends on the model. We also noticed that several loops with thermal flexibility show a positive NOE/ROE ratio that could be due to chemical exchange.

It is important to note the absence of any hydroxyl group in the vicinity of the residue 24 amide. A hydroxyl near the amide hydrogen could generate the NOE and ROE observed for Asp24 in Figure 5. Clearly, this case does not hold, as the nearest hydroxyl is approximately $7 \AA$ away and no NOE/ROE ratio was observed for the $\mathrm{D} 24 \mathrm{~N}$ mutant.

For the D24N mutant, we observed neither NOE nor ROE cross-peaks between water and the Asn 24 amide hydrogen. The absence of nuclear spin dipolar interaction could be due to the higher permeability of Asn24 in the water cavity, with water molecules exchanging too fast to build up the NOE/ROE ratio in such a short mixing time $(20 \mathrm{~ms})$. The other possibility would be the complete closing of the water cavity, becoming completely impermeable to water. We will show next, on the basis of molecular dynamics simulation, that the higher permeability is a more plausible explanation. By high permeability, we mean the increased accessibility of water to Asn24.

The data presented so far support the idea that yTrx 1 reaches a conformational equilibrium between conformational states that differ in the hydration of the water cavity. To provide more insight and support this view, we perturbed the conformational equilibrium by adding a subdenaturant concentration of urea. The result is very clear and shows that the addition of urea caused major chemical shift changes in the residues facing the water cavity, for the reduced and oxidized states (Figure 6). It is remarkable that the residues involved in the chemical shift changes elicited by the presence of urea are almost the same residues that are in conformational exchange as shown in Figures 1 and 2.

For more information about the hydration of the water cavity and the effect of the D24N mutation, we performed MD simulations of $y \operatorname{Trx} 1$ and $\mathrm{D} 24 \mathrm{~N}$ in the oxidized and reduced states (Figure 7). We simulated the proteins for $200 \mathrm{~ns}$, starting from the solution structure for the reduced conformation ${ }^{10}$ and 
A.
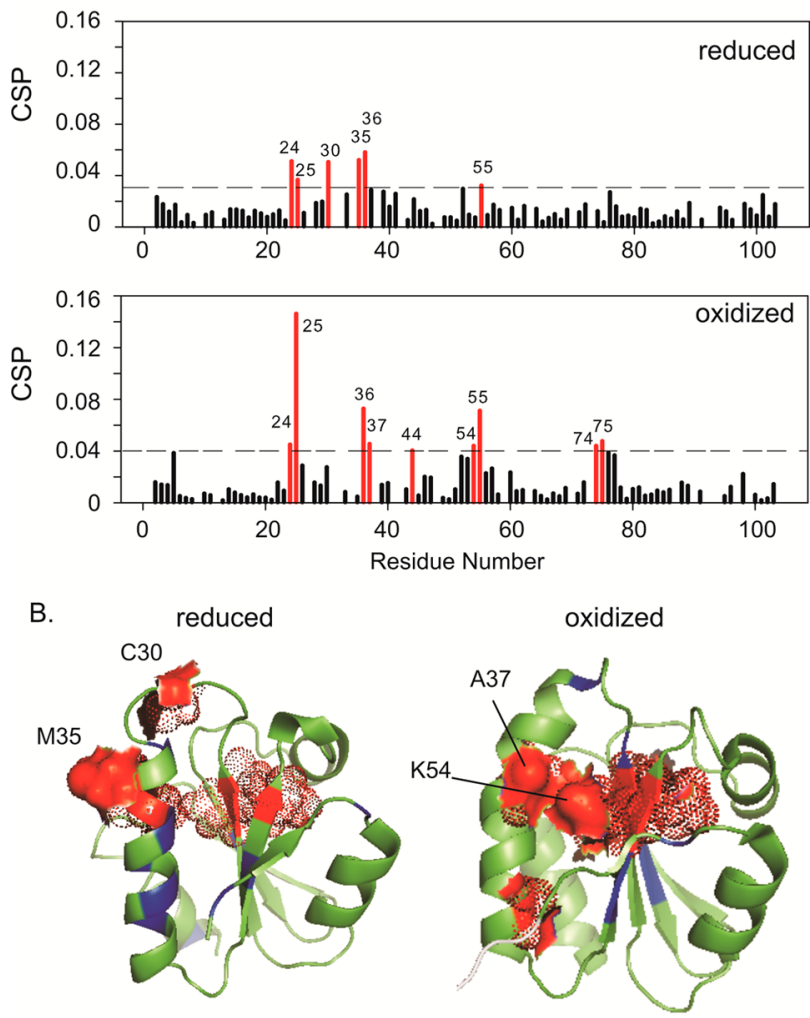

Figure 6. Effect of urea on the water cavity. (A) Chemical shift perturbation observed in the presence of subdenaturant concentrations of urea $(300 \mathrm{mM})$ for reduced and oxidized yTrx1. (B) Structure of the oxidized and reduced forms of yTrxl highlighted by chemical shift perturbation. The most prominent perturbations are colored red. All residues are internal for reduced and oxidized protein. They are the residues that form the water cavity. The residues facing the protein surface are represented as a surface plot, colored red (M35 and C30 for the reduced form and A37 and K54 for the oxidized form).
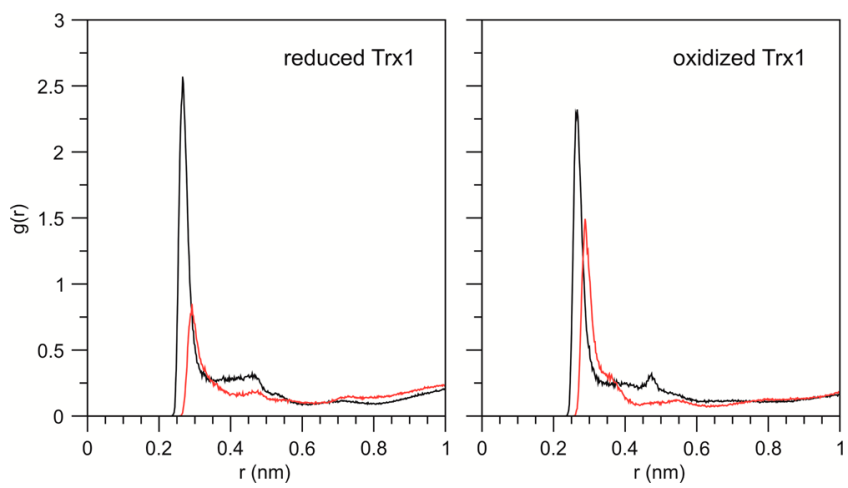

Figure 7. Asp/Asn 24 hydration state in $\mathrm{MD}$ simulations. Radial distribution function $[g(r)]$ of water molecules as a function of the carbonyl oxygen of the Asp/Asn24 side chains, for both the wild type (black line) and mutant D24N (red line), in the reduced and oxidized forms.

the crystal structure for the oxidized conformation. ${ }^{13}$ The D24N mutation was constructed in silico. The structure was stable throughout the $\mathrm{MD}$ simulation, and the hydration of D24 was analyzed.

The radial distribution functions (RDFs) for water molecules relative to residue 24 for yTrx 1 in the reduced and oxidized conformations are very similar, with a slight decrease in the probability of finding water for the oxidized state (Figure 6, black lines at the top, A and B). The highest-probability peak is at $2.7 \AA$, a distance that is compatible with the hydrogen bond between water and the oxygen of Asp24. The second peak is at $\sim 4.6 \AA$ and corresponds to the second layer of water, as seen in the crystal structure shown in Figure 5B. After the second layer, the probability distribution becomes more diffuse as it reaches the surface of the protein.

We observed a significant decrease in RDF values for D24N, meaning a consistently lower probability of finding water molecules inside the water cavity [peaks 1 and 2 (Figure 6)]. Even with the decreased probability, it is still possible to find water directly hydrogen-bonded to the Asn 24 side chain (peak $1)$, but on average at a distance $(2.9 \AA)$ greater than that seen for the wild-type Asp24. The probability of finding water molecules above $5 \AA$ is the same as that observed for wt yTrxl.

To understand the location of the internal water in yTrxl, we analyzed the presence of cavities in the Trxl structures using 20000 structures extracted from the MD simulations (Figure 8 ). The detection mode filters an ensemble of $\alpha$-spheres on a
A

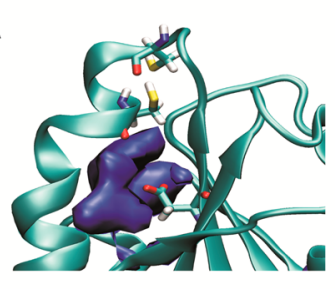

C

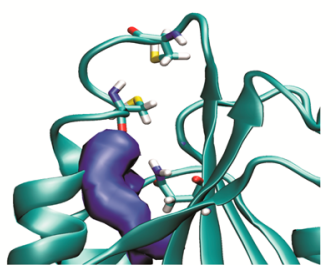

B

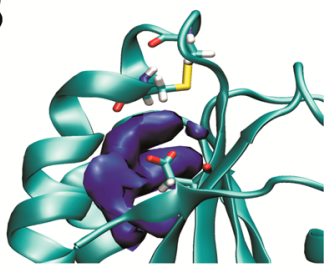

D

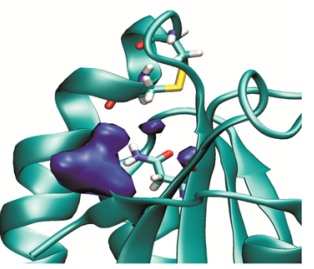

Figure 8. Water cavity in wt yTrx1 and its D24N mutant. Panels A and $\mathrm{C}$ show the reduced conformations of wt yTrx1 and its D24N mutant, respectively. Panels $\mathrm{B}$ and $\mathrm{D}$ show the oxidized conformations of wt yTrx1 and its D24N mutant, respectively. Active site residues Asp/ Asn24, Cys30, and Cys33 are represented as sticks, and the water cavity is represented as a solid blue surface. We used Fpocket ${ }^{48}$ to compute the cavities over the $200 \mathrm{~ns}$ MD simulation. To compute the isosurface, we used the same isovalue of 2.2 for the four simulations.

$\mathrm{MD}$ trajectory, allowing the location of intermediate size $\alpha$ spheres, which are typical of cavities. In this way, it is possible to define cavities that are representative of a MD trajectory.

The shape and size of the cavity for the wild type in the reduced and oxidized conformations are similar. The average cavity volume was approximately $300 \AA^{3}$, which can fit up to approximately 10 water molecules. When observing the presence of water within the cavity along the MD trajectory, we observed the formation of clusters of water molecules, varying from one to five water molecules. Most of the time the cavity was filled with three water molecules, for the reduced and oxidized states (Table S1 of the Supporting Information). The existence of a fully empty cavity was extremely rare (Table S1 of the Supporting Information).

In the reduced and oxidized D24N mutant, we observed a significant decrease in volume. The shape of the cavity also changed. The cavity became more superficial, decreasing the 
probability of entrance of a more internalized water molecule. For the reduced and oxidized states, the average volume decreased from $\sim 300$ to $\sim 200 \AA^{3}$. The cavity is open to entering water near Asp24 but almost closed near Cys33. The internal portion of the cavity, near Pro73, was closed in the mutant. For the reduced state, the volume was in the range of $100-400 \mathrm{~nm}^{3}$ at the beginning of the MD simulation and decreased to the range of $50-300 \mathrm{~nm}^{3}$ after MD simulation for $130 \mathrm{~ns}$. The more prevalent number of water molecules within the cavity is zero for the reduced state and one of the oxidized state (Table S1 of the Supporting Information).

Cavity detection is an important way to detect an empty space that is most likely occupied by water. However, to actually measure the entrance of water molecules within the cavity, we counted the number of long-lived hydrogen bonds between water and the Asp/Asn24 side chain (Table 1).

Table 1. Numbers of Water Molecules Hydrogen-Bonded to Asp/Asn24 in the MD Simulations of Reduced and Oxidized States

\begin{tabular}{ccccc} 
& \multicolumn{4}{c}{ no. of H-bonds } \\
\cline { 2 - 5 } $\begin{array}{c}\text { H-bond lifetime } \\
\text { (ns) }\end{array}$ & $\begin{array}{c}\text { reduced } \\
\text { yTrx1 }\end{array}$ & $\begin{array}{c}\text { reduced } \\
\text { D24N }\end{array}$ & $\begin{array}{c}\text { oxidized } \\
\text { yTrx1 }\end{array}$ & $\begin{array}{c}\text { oxidized } \\
\text { D24N }\end{array}$ \\
$1-3$ & 155 & 17 & 123 & 66 \\
$3-7$ & 49 & 4 & 40 & 11 \\
$7-11$ & 7 & 0 & 3 & 3 \\
$11-15$ & 6 & 0 & 3 & 3 \\
$15-19$ & 3 & 0 & 1 & 1 \\
$>19$ & 0 & 0 & 2 & 3 \\
total & 220 & 21 & 172 & 87 \\
\hline
\end{tabular}

We counted the number of long-lived water molecules hydrogen-bonded to the Asp/Asn 24 side chain during the entire MD simulations (Table 1). We defined long-lived as a hydrogen bond whose residence time was $>1$ ns. We observed that water has easy access to the cavity and thus to Asp/Asn24. During the MD simulations, thousands of water molecules were able to form short-lived hydrogen bonds with Asp/Asn24. This result is compatible with the previous observation of the formation of clusters of water at the water cavity. However, only a few water molecules were able to go deeper into the water cavity, forming long-lived hydrogen bonds.

Wild-type yTrx1 was able to hold a significant number of long-lived hydrogen-bonded water molecules, 220 for the reduced state and 172 for the oxidized state (Table 1). For the D24N mutant, the number of long-lived water molecules decreased considerably, to 21 for the reduced state $(9.5 \%)$ and 87 for the oxidized state (50\%). This result is compatible with the observation that the cavity is smaller and more superficial for the mutant. D24N is more permeable to water than the wild type, but on the other hand, water has only superficial access to the water cavity.

\section{DISCUSSION}

One of the ancestral features of thioredoxins is the presence of a water cavity. ${ }^{1-3}$ In this work, we measured the hydration and dynamics of yeast thioredoxin 1. The conserved aspartic acid buried in the hydrophobic core of the protein is a key residue in the modulation of dynamics. The catalytic role of this aspartic acid, as a proton acceptor in the reduction of the protein target, was very elegantly proposed as one of the mechanisms for the activation of the internal cysteine, during the 1980s and 1990s. ${ }^{2,18,58-60}$ Here, we propose a complementary role of coupling hydration and conformational motion of the water cavity and interacting loops. There is a conserved intimate contact between the water cavity and the interacting loops. This structural feature allows the possibility that motion within the water cavity affects the interacting loops and vice versa.

The D24N mutant quenches the conformational equilibrium observed for wt yTrx1 stabilizing a conformational state in which Asn24 is more accessible to water than wt Asp24. The water cavity becomes smaller and more superficial. The conformational state observed for $\mathrm{D} 24 \mathrm{~N}$ may provide insights into a possible relationship between the observed conformational equilibrium and catalysis. Four points contribute to this rationale. (i) The mutation significantly changes the dynamics of yTrx1, and the conformational dynamics was quenched for the residues involved in the inner cavity. (ii) The mutation stabilized a conformation in which $\mathrm{H}^{+}$exchange may be facilitated, because Asn24 is more exposed to water than Asp24 in the wild type. (iii) The mutation mimics the $\mathrm{H}^{+}$-associated form of Asp24 (-COOH vs $-\mathrm{CONH}_{2}$ ). (iv) It is well established that the ionization state of the buried Asp is important for the catalysis and stability of thioredoxins, which will be discussed below. Furthers studies are necessary to show that the conformational state stabilized by the $\mathrm{D} 24 \mathrm{~N}$ mutation is visited during the conformational equilibrium of wt yTrx1.

The dynamics of several thioredoxins have been described previously, $^{61-67}$ but for now, the relationship between the dynamic properties and their catalytic activity and interaction with multiple targets is not well-established. The reduced and oxidized forms of many species exhibit very similar dynamic behavior on the picosecond to nanosecond time scale, the same behavior observed here. The more pronounced differences are observed in motions on the micro- to millisecond time scale. Upon reanalysis of the $R_{2} / R_{1}$ ratio of $E$. coli $\operatorname{Trx},{ }^{68}$ we observed the presence of conformational exchange in the Asp26-Ile38 water cavity pair in the oxidized form, equivalent to the observed dynamics in the Asp24-Ile36 pair observed for yTrx1. Similar behavior was observed for the reduced form, where Ile38, a residue of the water cavity, is in conformational exchange (Ile38). There are also micro- to millisecond motions in the interacting loops. Unpublished data from our lab show the same dynamical behavior for yTrx $2 .^{11}$ The slow dynamics of the buried aspartic acid (Asp23) and other residues of the water cavity have also been shown for Trx of Alcyclobacillus acidocaldarius. ${ }^{62}$ On the basis of these observations, we cannot generalize the findings of this work but emphasize the importance of micro- to millisecond motions, inter-relating the water cavity and the interacting loops found for many thioredoxins.

Conformational equilibrium is a key step in thioredoxin activity. The conformational energy is the driving force for the reduction, in which the oxidized conformation is more stable than the reduced form. Another important point is that the protonation state of the buried aspartate finely tunes the stability of the protein. It has been shown for E. coli thioredoxin that the $\Delta G^{\circ}$ of unfolding increases from 8.6 to $10.6 \mathrm{kcal} / \mathrm{mol}$ upon the protonation of Asp26, meaning that the protonation of Asp26 stabilizes the protein. The D26A mutation stabilizes the protein, leading to a $\Delta G^{\circ}$ of unfolding of $13.2 \mathrm{kcal} / \mathrm{mol}{ }^{18}$ The conformational equilibrium at the water cavity of $y \operatorname{Trx} 1$ and $\mathrm{D} 24 \mathrm{~N}$ may be in great part governed by the differences in the solvation free energy of the side chains of an aspartate $(-82.57$ and $-80.65 \mathrm{kcal} / \mathrm{mol})$ and asparagines $(-17.47$ and 
$-9.70 \mathrm{kcal} / \mathrm{mol}){ }^{69}$ where the first value corresponds to the calculated and the second to the experimental solvation free energy, respectively. Solvation free energies of Asn are similar to those of the neutral Asp. The energetic cost for the desolvation of the aspartate anion is significantly greater than that for asparagine. Therefore, the total energy of the system is a balance between the conformational energy and the solvation energy. The opening-closing equilibrium of the water cavity changes the overall energy of the system, and the desolvation of the buried aspartic acid contributes significantly to the overall energy.

The $\mathrm{pH}$ titration of thioredoxins ${ }^{29,30,58}$ revealed many of the aspects of conformational equilibrium in thioredoxins and contributed significantly to the understanding of the catalytic mechanism. Many authors described complex titration curves $^{30,70}$ for the residues at the catalytic site, in particular the two cysteines and the buried aspartic acid. Multiple $\mathrm{p} K_{\mathrm{a}}$ values could be observed for each active site residues, as a result of coupling among several hydration and conformational events (electrostatic interactions ${ }^{71}$ ) at the catalytic site. It was also observed that several residues cotitrate with a $\mathrm{p} K_{\mathrm{a}}$ near 7.6, the value of the buried aspartic acid. ${ }^{29}$ A hydration-related event is proton sharing within the active site, ${ }^{28,29}$ and conformationrelated events would be the different orientations of the $\alpha$ helices, which leads to interaction of the helix dipole and the active site cysteines, ${ }^{28}$ modulating the $\mathrm{pK}_{\mathrm{a}}$ values of the cysteines and also specific hydrogen bonding between the active site amino acids. ${ }^{24,72}$ The coupling of hydration and conformational equilibrium is essential for thioredoxin catalysis and molecular recognition. All this motion is important for the prompt change in $\mathrm{p} K_{\mathrm{a}}$ upon binding, in which subangstrom positioning affects catalysis. ${ }^{21}$ Because Asn24, in the D24N mutant, mimics the protonated form of Asp24, this mutant may represent one of the catalytically important conformers, which was characterized here as a conformation that could facilitate $\mathrm{H}^{+}$exchange.

In conclusion, we showed that replacing Asp24 with Asn alters the hydration state of the water cavity and modulates conformational equilibria within yTrxl for the oxidized and reduced states. This suggests a correlation between the hydration state of Asp24 internal dynamics of yTrx1. The mutation of Asn24 consistently facilitates the desolvation necessary for closing of the inner part of the water cavity.

\section{ASSOCIATED CONTENT}

\section{S Supporting Information}

Raw relaxation data, extra information about the MD simulation, Lipari-Szabo modes, and proline cluster of the yTrx1 structure (Figures S1-S4 and Table S1). This material is available free of charge via the Internet at http://pubs.acs.org.

\section{AUTHOR INFORMATION}

\section{Corresponding Author}

*Av. Carlos Chagas Filho, 373, CCS, Annex CNRMN, Federal University of Rio de Janeiro, Rio de Janeiro, RJ, Brazil 21941902. E-mail: falmeida@cnrmn.bioqmed.ufrj.br. Phone: +552131042326.

\section{Author Contributions}

C.C.-S. and F.G.-N. contributed equally to this work.

\section{Funding}

We thank FAPERJ, CNPq, and CAPES for funding.

\section{Notes}

The authors declare no competing financial interest.

\section{ACKNOWLEDGMENTS}

We thank the NMR facility CNRMN (http://cnrmn.bioqmed. ufrj.br) for NMR time.

\section{ABBREVIATIONS}

NMR, nuclear magnetic resonance; Trx, thioredoxin; yTrx1, yeast thioredoxin 1; D24N, D24N mutant of yeast thioredoxin 1 ; CSP, chemical shift perturbation; RDF, radial distribution function.

\section{REFERENCES}

(1) LeMaster, D. M., Springer, P. A., and Unkefer, C. J. (1997) The role of the buried aspartate of Escherichia coli thioredoxin in the activation of the mixed disulfide intermediate. J. Biol. Chem. 272, 29998-30001.

(2) Dyson, H. J., Jeng, M. F., Tennant, L. L., Slaby, I., Lindell, M., Cui, D. S., Kuprin, S., and Holmgren, A. (1997) Effects of buried charged groups on cysteine thiol ionization and reactivity in Escherichia coli thioredoxin: Structural and functional characterization of mutants of Asp 26 and Lys 57. Biochemistry 36, 2622-2636.

(3) Ji, C., Mei, Y., and Zhang, J. Z. H. (2008) Developing polarized protein-specific charges for protein dynamics: $\mathrm{MD}$ free energy calculation of pKa shifts for Asp26/Asp20 in thioredoxin. Biophys. J. 95, 1080-1088.

(4) Arnér, E. S., and Holmgren, A. (2000) Physiological functions of thioredoxin and thioredoxin reductase. Eur. J. Biochem. 267, 61026109.

(5) Oliveira, M. A., Discola, K. F., Alves, S. V., Medrano, F. J., Guimarães, B. G., and Netto, L. E. S. (2010) Insights into the specificity of thioredoxin reductase-thioredoxin interactions. A structural and functional investigation of the yeast thioredoxin system. Biochemistry 49, 3317-3326.

(6) Potamitou, A., Holmgren, A., and Vlamis-Gardikas, A. (2002) Protein levels of Escherichia coli thioredoxins and glutaredoxins and their relation to null mutants, growth phase, and function. J. Biol. Chem. 277, 18561-18567.

(7) Holmgren, A., and Sengupta, R. (2010) The use of thiols by ribonucleotide reductase. Free Radical Biol. Med. 49, 1617-1628.

(8) Buchanan, B. B., Holmgren, A., Jacquot, J.-P., and Scheibe, R. (2012) Fifty years in the thioredoxin field and a bountiful harvest. Biochim. Biophys. Acta 1820, 1822-1829.

(9) Pinheiro, A. S., Amorim, G. C., Netto, L. E. S., Valente, A. P., and Almeida, F. C. L. (2006) ${ }^{1} \mathrm{H},{ }^{13} \mathrm{C}$ and ${ }^{15} \mathrm{~N}$ resonance assignments for the reduced forms of thioredoxin 1 and 2 from S. cerevisiae. J. Biomol. NMR 36 (Suppl. 1), 35.

(10) Pinheiro, A. S., Amorim, G. C., Netto, L. E. S., Almeida, F. C. L., and Valente, A. P. (2008) NMR solution structure of the reduced form of thioredoxin 1 from Sacharomyces cerevisiae. Proteins: Struct., Funct., Bioinf. 70, 584-587.

(11) Amorim, G. C., Pinheiro, A. S., Netto, L. E. S., Valente, A. P., and Almeida, F. C. L. (2007) NMR solution structure of the reduced form of thioredoxin 2 from Saccharomyces cerevisiae. J. Biomol. NMR 38, 99-104.

(12) Zhang, Y., Bao, R., Zhou, C.-Z., and Chen, Y. (2008) Expression, purification, crystallization and preliminary $\mathrm{X}$-ray diffraction analysis of thioredoxin Trx1 from Saccharomyces cerevisiae. Acta Crystallogr. F64, 323-325.

(13) Bao, R., Zhang, Y., Lou, X., Zhou, C.-Z., and Chen, Y. (2009) Structural and kinetic analysis of Saccharomyces cerevisiae thioredoxin Trx1: Implications for the catalytic mechanism of GSSG reduced by the thioredoxin system. Biochim. Biophys. Acta 1794, 1218-1223.

(14) Bao, R., Chen, Y., Tang, Y., Janin, J., and Zhou, C. (2007) Crystal Structure of the Yeast Cytoplasmic Thioredoxin Trx2. Bioinformatics 249, 246-249. 
(15) Ingles-Prieto, A., Ibarra-Molero, B., Delgado-Delgado, A., PerezJimenez, R., Fernandez, J. M., Gaucher, E. A., Sanchez-Ruiz, J. M., and Gavira, J. A. (2013) Conservation of Protein Structure over Four Billion Years. Structure, 1-8.

(16) Collet, J.-F., and Messens, J. (2010) Structure, function, and mechanism of thioredoxin proteins. Antioxid. Redox Signaling 13, $1205-1216$.

(17) Su, D., Berndt, C., Fomenko, D. E., Holmgren, A., and Gladyshev, V. N. (2007) A conserved cis-proline precludes metal binding by the active site thiolates in members of the thioredoxin family of proteins. Biochemistry 46, 6903-6910.

(18) Langsetmo, K., Fuchs, J. A., and Woodward, C. (1991) The conserved, buried aspartic acid in oxidized Escherichia coli thioredoxin has a pKa of 7.5. Its titration produces a related shift in global stability. Biochemistry 30, 7603-7609.

(19) Kallis, G., and Holmgren, A. (1980) Differential Reactivity of the Functional Sulfhydryl Groups of Cysteine-32 and Cysteine-35 Present in the reduced form of thioredoxin from Escherichia coli. J. Biol. Chem. 255, 10261-10265.

(20) Perez-Jimenez, R., Li, J., Kosuri, P., Sanchez-Romero, I., Wiita, A. P., Rodriguez-Larrea, D., Chueca, A., Holmgren, A., MirandaVizuete, A., Becker, K., Cho, S.-H., Beckwith, J., Gelhaye, E., Jacquot, J. P., Gaucher, E. A., Gaucher, E., Sanchez-Ruiz, J. M., Berne, B. J., and Fernandez, J. M. (2009) Diversity of chemical mechanisms in thioredoxin catalysis revealed by single-molecule force spectroscopy. Nat. Struct. Mol. Biol. 16, 890-896.

(21) Wiita, A. P., Perez-Jimenez, R., Walther, K. A., Gräter, F., Berne, B. J., Holmgren, A., Sanchez-Ruiz, J. M., and Fernandez, J. M. (2007) Probing the chemistry of thioredoxin catalysis with force. Nature 450, 124-127.

(22) Menchise, V., Corbier, C., Didierjean, C., Saviano, M., Benedetti, E., Jacquot, J., and Aubry, A. (2001) Crystal structure of the wild-type and D30A mutant thioredoxin $h$ of Chlamydomonas reinhardtii and implications for the catalytic mechanism. Biochem. J. $359,65-75$.

(23) Holmgren, A. (1995) Thioredoxin structure and mechanism: Conformational changes on oxidation of the active-site sulfhydryls to a disulfide. Curr. Biol. 2, 1-5.

(24) Roos, G., Foloppe, N., Van Laer, K., Wyns, L., Nilsson, L., Geerlings, P., and Messens, J. (2009) How Thioredoxin Dissociates Its Mixed Disulfide. PLoS Comput. Biol. 5, e1000461.

(25) Carvalho, A. T. P., Swart, M., van Stralen, J. N. P., Fernandes, P. A., Ramos, M. J., and Bickelhaupt, F. M. (2008) Mechanism of thioredoxin-catalyzed disulfide reduction. Activation of the buried thiol and role of the variable active-site residues. J. Phys. Chem. B 112, 2511-2523.

(26) Carvalho, A. T. P., Fernandes, P. A., and Ramos, M. J. (2006) Determination of the $\Delta \mathrm{pKa}$ between the active site cysteines of thioredoxin and DsbA. J. Comput. Chem. 27, 966-975.

(27) Cheng, Z., Zhang, J., Ballou, D. P., and Williams, C. H. (2011) Reactivity of thioredoxin as a protein thiol-disulfide oxidoreductase. Chem. Rev. 111, 5768-5783.

(28) Carvalho, A. T. P., Fernandes, P. A., and Ramos, M. J. (2006) Theoretical study of the unusual protonation properties of the active site cysteines in thioredoxin. J. Phys. Chem. B 110, 5758-5761.

(29) Jeng, M., Holmgren, A., and Dyson, H. J. (1995) Proton Sharing between Cysteine Thiols in Escherichia coli Thioredoxin: Implications for the Mechanism of Protein Disulfide Reduction. Biochemistry 34, 10101-10105.

(30) Chivers, P. T., Prehoda, K. E., Volkman, B. F., Kim, B. M., Markley, J. L., and Raines, R. T. (1997) Microscopic pKa values of Escherichia coli thioredoxin. Biochemistry 36, 14985-14991.

(31) Holmgren, A. (1979) Reduction of Disulfides by Thioredoxin. J. Biol. Chem. 254, 9113-9119.

(32) Sanz-Barrio, R., Millán, A. F.-S., Carballeda, J., Corral-Martínez, P., Seguí-Simarro, J. M., and Farran, I. (2011) Chaperone-like properties of tobacco plastid thioredoxins $\mathrm{f}$ and $\mathrm{m}$. J. Exp. Bot. 63, 365-379.
(33) Kern, R., Malki, A., Holmgren, A., and Richarme, G. (2003) Chaperone properties of Escherichia coli thioredoxin and thioredoxin reductase. Biochem. J. 371, 965-972.

(34) Delaglio, F., Grzesiek, S., Vuister, G. W., Zhu, G., Pfeifer, J., and Bax, A. (1995) Nmrpipe: A Multidimensional Spectral Processing System Based on Unix Pipes. J. Biomol. NMR 6, 277-293.

(35) Johnson, B. A., and Blevins, R. A. (1994) Nmr View: A Computer-Program for the Visualization and Analysis of NMR Data. J. Biomol. NMR 4, 603-614.

(36) Lipari, G., and Szabo, A. (1982) Model-Free Approach to the Interpretation of Nuclear Magnetic Resonance Relaxation in Macromolecules. 1. Theory and Range of Validity. J. Am. Chem. Soc. 104, 4546-4559.

(37) Lipari, G., and Szabo, A. (1982) Model-Free Approach to the Interpretation of Nuclear Magnetic Resonance Relaxation in Macromolecules. 2. Analysis of Experimental Results. J. Am. Chem. Soc. 104, $4559-4570$.

(38) Dosset, P., Hus, J. C., Blackledge, M., and Marion, D. (2000) Efficient analysis of macromolecular rotational diffusion from heteronuclear relaxation data. J. Biomol. NMR 16, 23-28.

(39) Li, H., Robertson, A. D., and Jensen, J. H. (2005) Very fast empirical prediction and rationalization of protein $\mathrm{pK}_{\mathrm{a}}$ values. Proteins: Struct., Funct., Bioinf. 61, 704-721.

(40) Schwieters, C. D., and Clore, G. M. (2001) The VMD-XPLOR visualization package for NMR structure refinement. J. Magn. Reson. 149, 239-244.

(41) Berendsen, H. J. C., Vanderspoel, D., and Vandrunen, R. (1995) Gromacs: A Message-Passing Parallel Molecular-Dynamics Implementation. Comput. Phys. Commun. 91, 43-56.

(42) Essmann, U., Perera, L., Berkowitz, M. L., Darden, T., Lee, H., and Pedersen, L. G. (1995) A smooth particle mesh Ewald method. J. Chem. Phys. 103, 8577-8593.

(43) Jorgensen, W. L., and Tiradorives, J. (1988) The Opls Potential Functions for Proteins: Energy Minimizations for Crystals of CyclicPeptides and Crambin. J. Am. Chem. Soc. 110, 1657-1666.

(44) Hess, B., Bekker, H., Berendsen, H. J. C., and Fraaije, J. (1997) LINCS: A linear constraint solver for molecular simulations. J. Comput. Chem. 18, 1463-1472.

(45) Miyamoto, S., and Kollman, P. A. (1992) SETTLE: An analytical version of the shake and rattle algorithm for rigid water models. J. Comput. Chem. 13, 952-962.

(46) Hornak, V., Abel, R., Okur, A., Strockbine, B., Roitberg, A., and Simmerling, C. (2006) Comparison of multiple Amber force fields and development of improved protein backbone parameters. Proteins 65 , $712-725$.

(47) MacKerell, A. D., Bashford, D., Bellott, M., Dunbrack, R. L., Evanseck, J. D., Field, M. J., Fischer, S., Gao, J., Guo, H., Ha, S., Joseph-McCarthy, D., Kuchnir, L., Kuczera, K., Lau, F. T. K., Mattos, C., Michnick, S., Ngo, T., Nguyen, D. T., Prodhom, B., Reiher, W. E., Roux, B., Schlenkrich, M., Smith, J. C., Stote, R., Straub, J., Watanabe, M., Wiorkiewicz-Kuczera, J., Yin, D., and Karplus, M. (1998) All-atom empirical potential for molecular modeling and dynamics studies of proteins. J. Phys. Chem. B 102, 3586-3616.

(48) Le Guilloux, V., Schmidtke, P., and Tuffery, P. (2009) Fpocket: An open source platform for ligand pocket detection. BMC Bioinf. 10, 168.

(49) Holmgren, A., and Lu, J. (2010) Thioredoxin and thioredoxin reductase: Current research with special reference to human disease. Biochem. Biophys. Res. Commun. 396, 120-124.

(50) Rubini, M., Schärer, M. A., Capitani, G., and Glockshuber, R. (2013) (4R)- and (4S)-fluoroproline in the conserved cis-prolyl peptide bond of the thioredoxin fold: Tertiary structure context dictates ring puckering. ChemBioChem 14, 1053-1057.

(51) Otting, G., Liepinsh, E., and Wüthrich, K. (1991) Protein hydration in aqueous solution. Science 254, 974-980.

(52) Otting, G., Liepinsh, E., Farmer, B. T., and Wüthrich, K. (1991) Protein hydration studied with homonuclear $3 \mathrm{D}{ }^{1} \mathrm{H}$ NMR experiments. J. Biomol. NMR 1, 209-215. 
(53) Modig, K., Liepinsh, E., Otting, G., and Halle, B. (2004) Dynamics of Protein and Peptide Hydration. Methods 126, 102-114. (54) Weichsel, A., Kem, M., and Montfort, W. R. (2010) Crystal structure of human thioredoxin revealing an unraveled helix and exposed S-nitrosation site. Protein Sci. 19, 1801-1806.

(55) Campos-Acevedo, A. A., Garcia-Orozco, K. D., Sotelo-Mundo, R. R., and Rudiño-Piñera, E. (2013) Expression, purification, crystallization and X-ray crystallographic studies of different redox states of the active site of thioredoxin 1 from the whiteleg shrimp Litopenaeus vannamei. Acta Crystallogr. F69, 488-493.

(56) Bao, R., Zhang, Y., Zhou, C.-Z., and Chen, Y. (2009) Structural and mechanistic analyses of yeast mitochondrial thioredoxin $\operatorname{Trx} 3$ reveal putative function of its additional cysteine residues. Biochim. Biophys. Acta 1794, 716-721.

(57) Modig, K., Liepinsh, E., Otting, G., and Halle, B. (2004) Dynamics of protein and peptide hydration. J. Am. Chem. Soc. 126, 102-114.

(58) Wilson, N. A., Barbar, E., Fuchs, J. A., and Woodward, C. (1995) Aspartic Acid 26 in Reduced Escherichia coli Thioredoxin Has a PKa $>9$. Biochemistry 34, 8931-8939.

(59) Jeng, M., and Dyson, H. J. (1996) Direct Measurement of the Aspartic Acid 26 pKa for Reduced Escherichia coli. Biochemistry 35, 16.

(60) Setterdahl, A. T., Chivers, P. T., Hirasawa, M., Lemaire, S. D., Keryer, E., Miginiac-Maslow, M., Kim, S.-K., Mason, J., Jacquot, J.-P., Longbine, C. C., de Lamotte-Guery, F., and Knaff, D. B. (2003) Effect of $\mathrm{pH}$ on the oxidation-reduction properties of thioredoxins. Biochemistry 42, 14877-14884.

(61) Kelley, J. J., Caputo, T. M., Eaton, S. F., Laue, T. M., and Bushweller, J. H. (1997) Comparison of backbone dynamics of reduced and oxidized Escherichia coli glutaredoxin-1 using ${ }^{15} \mathrm{~N}$ NMR relaxation measurements. Biochemistry 36, 5029-5044.

(62) Leone, M., Di Lello, P., Ohlenschläger, O., Pedone, E. M., Bartolucci, S., Rossi, M., Di Blasio, B., Pedone, C., Saviano, M., Isernia, C., and Fattorusso, R. (2004) Solution structure and backbone dynamics of the K18G/R82E Alicyclobacillus acidocaldarius thioredoxin mutant: A molecular analysis of its reduced thermal stability. Biochemistry 43, 6043-6058.

(63) Peterson, F. C., Lytle, B. L., Sampath, S., Vinarov, D., Tyler, E., Shahan, M., Markley, J. L., and Volkman, B. F. (2005) Solution structure of thioredoxin h1 from Arabidopsis thaliana. Protein Sci. 14, 2195-2200.

(64) Stone, M. J., Chandrasekhar, K., Holmgren, A., Wright, P. E., and Dyson, H. J. (1993) Comparison of backbone and tryptophan side-chain dynamics of reduced and oxidized Escherichia coli thioredoxin using ${ }^{15} \mathrm{~N}$ NMR relaxation measurements. Biochemistry $32,426-435$.

(65) LeMaster, D. M., and Kushlan, D. M. (1996) Dynamical Mapping of E. coli Thioredoxin via ${ }^{13} \mathrm{C}$ NMR Relaxation Analysis. J. Am. Chem. Soc. 118, 9255-9264.

(66) Lancelin, J. M., Guilhaudis, L., Krimm, I., Blackledge, M. J., Marion, D., and Jacquot, J. P. (2000) NMR structures of thioredoxin $\mathrm{m}$ from the green alga Chlamydomonas reinhardtii. Proteins 41, 334349.

(67) Dangi, B., Dobrodumov, A. V., Louis, J. M., and Gronenborn, A. M. (2002) Solution structure and dynamics of the human-Escherichia coli thioredoxin chimera: Insights into thermodynamic stability. Biochemistry 41, 9376-9388.

(68) Stone, M. J., Chandrasekhar, K., Holmgren, A., Wright, P. E., and Dyson, H. J. (1993) Comparison of backbone and tryptophan side-chain dynamics of reduced and oxidized Escherichia coli thioredoxin using nitrogen-15 NMR relaxation measurements. Biochemistry 32, 426-435.

(69) Dixit, S. B., Bhasin, R., Rajasekaran, E., and Jayaram, B. (1997) Solvation thermodynamics of amino acids. Assessment of the electrostatic contribution and force-field dependence. J. Chem. Soc., Faraday Trans. 93, 1105-1113.
(70) Chivers, P. T., and Raines, R. T. (1997) General acid/base catalysis in the active site of Escherichia coli thioredoxin. Biochemistry $36,15810-15816$.

(71) Warshel, A., and Aqvist, J. (1991) Electrostatic energy and macromolecular function. Annu. Rev. Biophys. Biophys. Chem. 20, 267298.

(72) Roos, G., Geerlings, P., and Messens, J. (2010) The conserved active site tryptophan of thioredoxin has no effect on its redox properties. Protein Sci. 19, 190-194. 